\title{
Posterior reversible encephalopathy syndrome as a neuropsychiatric manifestation of systemic lupus erythematosus
}

\author{
CM Ho *, MB, ChB, MRCP, CC Mok, MD, FRCP (Lond) (Edin) \\ Department of Medicine and Geriatrics, Tuen Mun Hospital, Tuen Mun, Hong Kong \\ *Corresponding author: hocm283@gmail.com \\ Hong Kong Med J 2019;25:410-2 \\ https://doi.org/10.12809/hkmj187579
}

\section{Introduction}

Neuropsychiatric manifestations of systemic lupus erythematosus (SLE) are heterogeneous and complex. Posterior reversible encephalopathy syndrome (PRES) and its association with SLE has increasingly been recognised ${ }^{1-4}$ In the 1999 American College of Rheumatology nomenclature for neuropsychiatric SLE, PRES is not classified as a distinct primary syndrome. ${ }^{5}$ We recently encountered a patient with active SLE who developed PRES. Given the strong association between PRES and active SLE, and the recent evidence for an inflammatory aetiology, we believe PRES should be re-evaluated as a possible primary neuropsychiatric SLE syndrome.

\section{Posterior reversible encephalopathy syndrome and systemic lupus erythematosus}

In 1996, Hinchey et $\mathrm{al}^{6}$ first described PRES as a reversible brain syndrome with typical clinical and radiological finding. Common presentations include headache, vomiting, altered mental function, visual symptoms, and seizures. ${ }^{1,2}$ Diagnosis is supported by classical symptoms and typical radiological feature of bilateral posterior subcortical brain oedema on magnetic resonance imaging (MRI). ${ }^{1}$ Classically, PRES is linked with accelerated hypertension, renal impairment, eclampsia, pre-eclampsia, sepsis, cytotoxic therapy, underlying autoimmune disease, and immunosuppressive therapy. ${ }^{1}$ After Hinchey el $\mathrm{al}^{6}$ first described PRES in 1996, strong evidence for a link between PRES and SLE has been established. In one case series of 120 patients with PRES, 18\% were diagnosed with SLE. ${ }^{1}$ Another recent study from Taiwan involving 3746 patients with SLE described a $0.69 \%$ prevalence of PRES episodes. ${ }^{2}$

Patients with active SLE who are receiving immunosuppressants are at risk of developing PRES. A Korean case series reported 15 patients with SLE who developed PRES, $80 \%$ of whom had renal insufficiency (serum creatinine $\geq 132.6 \mu \mathrm{mol} / \mathrm{L}$ ) that was associated with a 129 -fold increased risk of this complication. Other risk factors for PRES included hypertension, current treatment with high-dose steroids or cyclophosphamide, blood transfusion, hypoalbuminaemia, and high SLE Disease Activity Index. ${ }^{3}$ Other immunosuppressants such as calcineurin inhibitors, mycophenolate, and rituximab have also been implicated in the development of PRES. ${ }^{6,7}$ However, the association between PRES and medication use should be interpreted with caution because patients receiving these drugs usually have high background SLE disease activity. More recently, hyperlipidaemia and lymphopenia have been described as risk factors for PRES in patients with SLE. ${ }^{8}$

It is important to differentiate PRES from other active neuropsychiatric manifestations of SLE because treatment of PRES alone does not require immunosuppression. Other differential diagnoses of neuropsychiatric symptoms of SLE such as central nervous systemic infection, cerebrovascular events, metabolic and electrolyte disturbances, and adverse drug reactions must be excluded. A high index of suspicion is needed to diagnose PRES. Typical features of headache, acute hypertension, seizures, visual symptoms, and altered mental state should be recognised. Urgent MRI is the standard imaging study to diagnose PRES. Prompt treatment of PRES is necessary to prevent permanent neurological damage. Usually, PRES is completely reversible with blood pressure control and supportive measures; immunosuppression is indicated only for treating the underlying active SLE.

\section{Pathophysiology of posterior reversible encephalopathy syndrome in systemic lupus erythematosus}

The pathophysiology of PRES in SLE remains unclear. The classical understanding is that an acute rise in blood pressure exceeds the autoregulation of cerebral circulation, leading to increased cerebral blood flow and hyperperfusion brain injury. On the contrary, an excessive autoregulation response may cause a focal cerebral vasoconstriction, leading to brain ischaemia. The attribution to hypertensive crisis may explain part of the pathophysiology. 
However, the mechanism of PRES is unlikely explained by hypertension alone. Several cases of PRES in patients with SLE with normal blood pressure have been reported. ${ }^{3,6,9}$ Emerging evidence has shown that PRES in patients with SLE may be influenced by inflammatory mechanisms. ${ }^{10,11}$

Interleukin 6 plays an important role in the inflammatory process in neuropsychiatric SLE. Previous studies had already revealed an elevated cerebrospinal fluid interleukin 6 level in neuropsychiatric patients with SLE. ${ }^{12}$ Recently, a study on patients with SLE with PRES found a 2.84fold increase in serum interleukin 6 level compared with control patients with active SLE without PRES. ${ }^{11}$ Interleukin 6 activates the STAT-3 pathway and upregulates the expression of ICAM-1, VCAM- 1 , and endothelial nitric oxide synthase. ${ }^{13}$ These molecules activate the endothelium of blood vessels, increasing vascular permeability. In a normal physiological state, this mechanism allows cell migration for normal inflammatory processes. In PRES, the endothelial dysfunction and disruption of the bloodbrain barrier predispose patients to hyperperfusioninduced vasogenic oedema and neurological damage.

\section{Exemplar case}

A 24-year-old woman with known SLE since childhood was admitted to Tuen Mun Hospital, Hong Kong, in February 2018 for a serious disease flare with profound cytopenia and worsening lupus nephritis. The patient's SLE had been diagnosed in 2006 when she presented with haemolytic anaemia and diffuse global lupus nephritis (International Society of Nephrology/Renal Pathology Society class IVG). She had tested positive for anti-nuclear antibody screening, anti-double stranded DNA, anti-Ro, and anti-cardiolipin antibodies. The patient's SLE had become unstable in the past 2 years with multiple episodes of disease relapse involving haematological and renal systems. She became glucocorticoid dependent and had received several treatment modalities, including intravenous immunoglobulin, mycophenolate mofetil, cyclophosphamide, tacrolimus, and rituximab.

On presentation, the patient had severe thrombocytopenia $\left(38 \times 10^{9} / \mathrm{L}\right)$, haemolytic anaemia (haemoglobin $69 \mathrm{~g} / \mathrm{L}$ ), serositis, acute renal function deterioration, and proteinuria (urine protein/ creatinine ratio 4.38). Because her cytopenia and kidney disease was refractory to high-dose glucocorticoid and mycophenolic acid, another course of intravenous immunoglobulin and rituximab was given on 8 March 2018. The patient developed headache on 16 March 2018 but a computed tomography (CT) scan of the brain was normal. Her renal function further deteriorated, with serum creatinine level $217 \mu \mathrm{mol} / \mathrm{L}$ (reference range, 50-98 $\mu \mathrm{mol} / \mathrm{L}$ ). The patient's blood pressure increased from her baseline of 130/80 $\mathrm{mm} \mathrm{Hg}$, recorded 1 day before seizure onset, to $150 / 100 \mathrm{~mm} \mathrm{Hg}$ on the day of seizure onset, shortly before she suddenly developed repeated episodes of tonic-clonic convulsions. She was transferred to the intensive care unit and treated with intravenous levetiracetam and propofol to control the status epilepticus and labetalol to control blood pressure. A new CT image of the brain showed a new hypo-attenuating area affecting the cortical and subcortical regions of bilateral occipital lobe (Fig a). An MRI image of the brain showed bilateral white-matter oedema with posterior and subcortical predominance (Fig b), which was compatible with

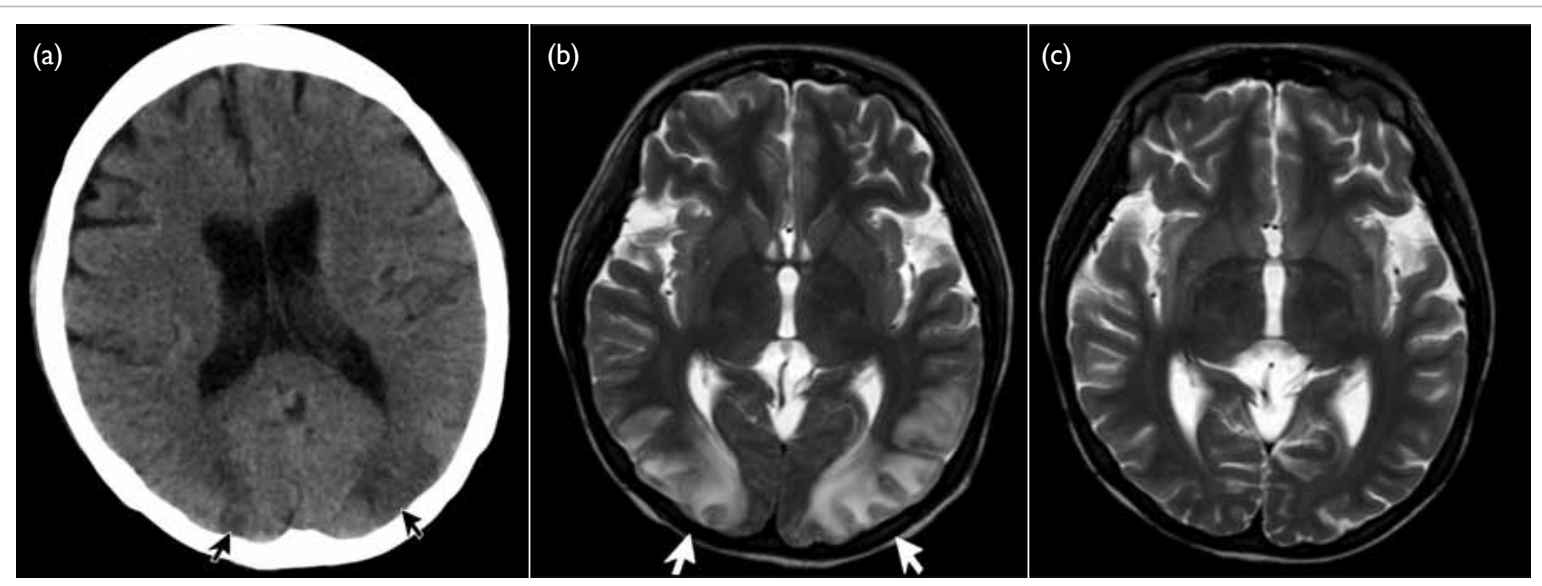

FIG. Diagnostic images of a 24-year-old woman with systemic lupus erythematosus presenting with headache who developed tonic-clonic seizures. (a) Computed tomography (CT) image of the brain on the day of the seizures showing bilateral occipital hypodense lesions (arrows) that were not present on earlier CT images. (b) Magnetic resonance image (MRI) of the brain on the day after the seizures showing bilateral posterior predominant T2 hyperintense vasogenic oedema (arrows). (c) New MRI of the brain at 3 months after the seizures showing complete resolution of the oedema 
PRES. After 5 days, the adequate control of the patient's seizures and blood pressure were achieved, and she was extubated. However, this was followed by transient confusion and visual hallucinations for the next 2 days before a full neurological recovery. Further doses of rituximab were given to salvage the patient's renal disease and cytopenia. There was no recurrence of the seizures and a complete resolution of the brain oedema on MRI image of the brain taken 3 months later (Fig c).

\section{Conclusions}

Prompt recognition and treatment of PRES is important in patients with SLE. The strong association of PRES with active SLE, particularly nephritis, and elevation of serum cytokines supports an inflammatory process that leads to endothelial dysfunction. In this sense, PRES should be reevaluated as a primary neuropsychiatric manifestation of SLE. The efficacy of immunosuppressive therapy in addition to blood pressure control and other supportive measures in PRES should be further evaluated in clinical trials.

\section{Author contributions}

$\mathrm{CM}$ Ho reviewed the case, extracted the patient's clinical information, and wrote the article. All authors performed literature research and critical revision for the important intellectual content. All authors had full access to the data, contributed to the study, approved the final version for publication, and take responsibility for its accuracy and integrity.

\section{Conflicts of interest}

The authors have no conflicts of interest to disclose.

\section{Funding/support}

This research received no specific grant from any funding agency in the public, commercial, or not-for-profit sectors.

\section{Ethics approval}

Informed consent was obtained from the patient.

\section{References}

1. Fugate JE, Claassen DO, Cloft HJ, Kallmes DF, Kozak OS, Rabinstein AA. Posterior reversible encephalopathy syndrome: associated clinical and radiologic findings. Mayo Clin Proc 2010;85:427-32.

2. Lai CC, Chen WS, Chang YS, et al. Clinical features and outcomes of posterior reversible encephalopathy syndrome in patients with systemic lupus erythematosus. Arthritis Care Res (Hoboken) 2013;65:1766-74.

3. Jung SM, Moon SJ, Kwok SK, et al. Posterior reversible encephalopathy syndrome in Korean patients with systemic lupus erythematosus: risk factors and clinical outcome. Lupus 2013;22:885-91.

4. Damrongpipatkul U, Oranratanachai K, Kasitanon N, Wuttiplakorn S, Louthrenoo W. Clinical features, outcome, and associated factors for posterior reversible encephalopathy in Thai patients with systemic lupus erythematosus: a case-control study. Clin Rheumatol 2018;37:691-702.

5. The American College of Rheumatology nomenclature and case definitions for neuropsychiatric lupus syndromes. Arthritis Rheum 1999;42:599-608.

6. Hinchey J, Chaves C, Appignani B, et al. A reversible posterior leukoencephalopathy syndrome. N Engl J Med 1996;334:494-500.

7. Mondal S, Goswami RP, Sinha D, et al. Posterior reversible encephalopathy syndrome in a patient with lupus nephritis on rituximab therapy: a challenge to find the offender. Lupus 2016;25:445-6.

8. Merayo-Chalico J, Apodaca E, Barrera-Vargas A, et al. Clinical outcomes and risk factors for posterior reversible encephalopathy syndrome in systemic lupus erythematosus: a multicentric case-control study. J Neurol Neurosurg Psychiatry 2016;87:287-94.

9. Kur JK, Esdaile JM. Posterior reversible encephalopathy syndrome-an underrecognized manifestation of systemic lupus erythematosus. J Rheumatol 2006;33:2178-83.

10. Marra A, Vargas M, Striano P, Del Guercio L, Buonanno P, Servillo G. Posterior reversible encephalopathy syndrome: the endothelial hypotheses. Med Hypotheses 2014;82:61922.

11. Merayo-Chalico J, Barrera-Vargas A, Juárez-Vega G, Alcocer-Varela J, Arauz A, Gómez-Martín D. Differential serum cytokine profile in patient with systemic lupus erythematosus and posterior reversible encephalopathy syndrome. Clin Exp Immunol 2018;192:165-70.

12. Fragoso-Loyo H, Richaud-Patin Y, Orozco-Narváez, et al. Interleukin-6 and chemokines in the neuropsychiatric manifestations of systemic lupus erythematosus. Arthritis Rheum 2007;56:1242-50.

13. Wei Z, Jiang W, Wang $H$, et al. The IL-6/STAT3 pathway regulates adhesion molecules and cytoskeleton of endothelial cells in thromboangiitis obliterans. Cell Signal 2018;44:118-26. 